\title{
Some Mixed Soft Operations and Extremally Soft Disconnectedness via Two Soft Topologies
}

\author{
Nihal Arabacioğlu Tas, Ahu Acikgoz \\ Department of Mathematics, Balikesir University, Balikesir, Turkey \\ Email: nihalarabacioglu@hotmail.com, ahuacikgoz@gmail.com
}

Received October 23, 2013; revised November 23, 2013; accepted November 30, 2013

Copyright (C) 2014 Nihal Arabacıoglu Tas, Ahu Acikgoz. This is an open access article distributed under the Creative Commons Attribution License, which permits unrestricted use, distribution, and reproduction in any medium, provided the original work is properly cited. In accordance of the Creative Commons Attribution License all Copyrights $\mathbb{C} 2014$ are reserved for SCIRP and the owner of the intellectual property Nihal Arabacioğlu Tas, Ahu Acikgoz. All Copyright (C) 2014 are guarded by law and by SCIRP as a guardian.

\section{ABSTRACT}

In this paper, we study the concept of soft sets which is introduced by Molodtsov [5]. We give the definition of $\left(\tau_{1}, \tau_{2}\right)$-semi open soft (resp. $\left(\tau_{1}, \tau_{2}\right)$-pre open soft, $\left(\tau_{1}, \tau_{2}\right)$ - $\alpha$-open soft, $\left(\tau_{1}, \tau_{2}\right)-\beta$-open soft, $\left(\tau_{1}, \tau_{2}\right)^{\prime}$-semi open soft, $\left(\tau_{1}, \tau_{2}\right)^{\prime}$-pre open soft, $\left(\tau_{1}, \tau_{2}\right)^{\prime}-\alpha$-open soft, $\left(\tau_{1}, \tau_{2}\right)^{\prime}-\beta$-open soft) set via two soft topologies. Also we introduce $\left(\tau_{1}, \tau_{2}\right)$-regular open soft and ESDC on two soft topologies. The aim of this paper is to investigate properties of some mixed soft operations and characterizations of ESDC. Finally, we study $\tau_{2}-E S D C$ soft topologies $\tau_{1}$.

\section{KEYWORDS}

$\left(\tau_{1}, \tau_{2}\right)$-Semi Open Soft; $\left(\tau_{1}, \tau_{2}\right)$-Pre Open Soft; $\left(\tau_{1}, \tau_{2}\right)-\alpha$-Open Soft; $\left(\tau_{1}, \tau_{2}\right)-\beta$-Open Soft; $\left(\tau_{1}, \tau_{2}\right)^{\prime}$-Semi Open Soft; $\left(\tau_{1}, \tau_{2}\right)^{\prime}$-Pre Open Soft; $\left(\tau_{1}, \tau_{2}\right)^{\prime}-\alpha$-Open Soft; $\left(\tau_{1}, \tau_{2}\right)^{\prime}-\beta$-Open Soft; $\left(\tau_{1}, \tau_{2}\right)$-Regular Open Soft; ESDC

\section{Introduction}

Some set theories can be dealt with unclear concepts, for example theory of rough sets and theory of fuzzy sets. Unfortunately, these theories are not sufficient to deal with some difficulties and encounter some problems. In 2009, Ali, Feng, Liu, Min and Shabir [1] investigated several operations on soft sets and introduced some new notions such as the restricted intersection, the restricted union etc. In 2011, Hussain and Ahmad [2] researched some properties of soft topological space. Kandil et al. [3] had defined semi-open (pre-open, $\alpha$-open, $\beta$ open) soft set and semi (pre, $\alpha, \beta$ )-soft continuity via these soft sets. In 2003, Maji, Biswas and Roy [4] introduced equality of two soft sets, subset of a soft set, null soft set, absolute soft set etc. In 1999, Molodtsov [5] introduced the soft theory as a general mathematical tool for dealing with these problems. He accomplished very significant applications of soft set theory such as solving some complications in economics, social science, medical science, engineering etc. In 2011, Shabir and Naz [6] defined and studied some notions such as soft topological space, soft interior, soft closure etc. In 2012, Zorlutuna et al. [7] introduced the concepts of soft interior point, soft interior, soft neighborhood, soft continuity and soft compactness. Later there has been an extensive study on the applications of soft set theory. Many people have studided soft theory and investigated some properties of this theory.

The aim of the present paper is to introduce and study notions of $\left(\tau_{1}, \tau_{2}\right)$-semi open soft (resp. $\left(\tau_{1}, \tau_{2}\right)$-pre open soft, $\left(\tau_{1}, \tau_{2}\right)$ - $\alpha$-open soft, $\left(\tau_{1}, \tau_{2}\right)$ - $\beta$-open soft, $\left(\tau_{1}, \tau_{2}\right)^{\prime}$-semi open soft, $\left(\tau_{1}, \tau_{2}\right)^{\prime}$-pre-open soft, 
$\left(\tau_{1}, \tau_{2}\right)^{\prime}-\alpha$-open soft, $\left(\tau_{1}, \tau_{2}\right)^{\prime}-\beta$-open soft) set via two soft topologies. For this purpose, we consider two soft topologies $\tau_{1}$ and $\tau_{2}$ over $X$. Also we define $\left(\tau_{1}, \tau_{2}\right)$-regular open soft and ESDC on two soft topologies. Furthermore, we investigate some properties of some mixed soft operations and some characterizations of ESDC.

Finally, we show $\tau_{2}$-ESDC soft topologies $\tau_{1}$.

\section{Preliminaries}

\section{Soft sets and Soft Topology}

Definition 2.1 [1]. The complement of a soft set $(F, A)$ is defined as $(F, A)^{c}=\left(F^{c}, A\right)$, where

$F^{c}(e)=(F(e))^{c}=X-F(e)$, for all $e \in A$.

Theorem 2.1 [2]. Let $(X, \tau, E)$ be a soft topological space over $X,(F, E)$ and $(G, E)$ are soft sets over $X$. Then

1) $\overline{\tilde{\varnothing}}=\tilde{\varnothing}$ and $\overline{\tilde{X}}=\tilde{X}$.

2) $(F, E) \tilde{\subseteq} \overline{(F, E)}$.

3) $(F, E)$ is a closed set if and only if $(F, E)=\overline{(F, E)}$.

4) $\overline{\overline{(F, E)}}=\overline{(F, E)}$.

5) $(F, E) \tilde{\subseteq}(G, E)$ implies $\overline{(F, E)} \tilde{\subseteq} \overline{(G, E)}$.

6) $\overline{(F, E) \cup(G, E)}=\overline{(F, E)} \cup \overline{(G, E)}$.

7) $\overline{(F, E) \cap(G, E)} \tilde{\subseteq} \overline{(F, E)} \cap \overline{(G, E)}$.

Theorem 2.2 [2]. Let $(X, \tau, E)$ be a soft topological space over $X$ and $(F, E)$ and $(G, E)$ are soft sets over $X$. Then

1) $\tilde{\varnothing}^{\circ}=\tilde{\varnothing}$ and $\tilde{X}^{o}=\tilde{X}$.

2) $(F, E)^{o} \tilde{\subseteq}(F, E)$.

3) $\left((F, E)^{o}\right)^{o}=(F, E)^{o}$.

4) $(F, E)$ is a soft open set if and only if $(F, E)^{\circ}=(F, E)$.

5) $(F, E) \tilde{\subseteq}(G, E)$ implies $(F, E)^{o} \tilde{\subseteq}(G, E)^{o}$.

6) $(F, E)^{o} \cap(G, E)^{o}=((F, E) \cap(G, E))^{o}$.

7) $(F, E)^{o} \cup(G, E)^{o} \tilde{(}((F, E) \cup(G, E))^{o}$.

Theorem 2.3 [2]. Let $(F, E)$ be a soft set of soft topological space over $X$. Then

1) $\left((F, E)^{c}\right)^{o}=(\overline{(F, E)})^{c}$.

2) $\overline{(F, E)^{c}}=\left((F, E)^{o}\right)^{c}$.

3) $(F, E)^{o}=\left(\overline{(F, E)^{c}}\right)^{c}$.

Definition 2.2 [3]. Let $(X, \tau, E)$ be a soft topological space and $(F, E) \in S S(X)_{E}$. Then $(F, E)$ is said to be

1) pre-open soft set if $(F, E) \tilde{\subseteq} \operatorname{int}(\operatorname{cl}(F, E))$;

2) semi-open soft set if $(F, E) \tilde{\subseteq} \operatorname{cl}(\operatorname{int}(F, E))$;

3) $\alpha$-open soft set if $(F, E) \tilde{\subseteq} \operatorname{int}(\operatorname{cl}(\operatorname{int}(F, E)))$;

4) $\beta$-open soft set if $(F, E) \subseteq \operatorname{cl}(\operatorname{int}(\operatorname{cl}(F, E)))$.

Definition 2.3 [4]. Let $(F, A)$ and $(G, B)$ be two soft sets over a common universe $X$. Then $(F, A)$ is said to be a soft subset of $(G, B)$ if $A \subseteq B$ and $F(e) \subseteq G(e)$, for all $e \in A$. This relation is denoted by $(F, A) \tilde{\subseteq}(G, B)$.

Definition 2.4 [4]. A soft set $(F, A)$ over $X$ is said to be a null soft set if $F(e)=\varnothing$, for all $e \in A$. This is denoted by $\tilde{\varnothing}$. 
Definition 2.5 [4]. A soft set $(F, A)$ over $X$ is said to be an absolute soft set if $F(e)=X$, for all $e \in A$. This denoted by $\tilde{X}$.

Definition 2.6 [4]. The union of two soft sets $(F, A)$ and $(G, B)$ over the common universe $X$ is the soft set $(H, C)$, where $C=A \cup B$ and $H(e)=F(e)$ if $e \in A-B$ or $H(e)=G(e)$ if $e \in B-A$ or $H(e)=F(e) \cup G(e)$ if $e \in A \cap B$ for all $e \in C$.

Definition 2.7 [4]. The intersection of two soft sets $(F, A)$ and $(G, B)$ over the common universe $X$ is the soft set $(H, C)$, where $C=A \cap B$ and for all $e \in C, H(e)=F(e) \cap G(e)$.

Definition 2.8 [5]. A pair $(F, A)$, where $F$ is mapping from $A$ to $P(X)$, is called a soft set over $X$. The family of all soft sets on $X$ is denoted by $S S(X)_{E}$.

$(F, A)$ is said to be soft equal to $(G, B)$ if $(F, A) \tilde{\subseteq}(G, B)$ and $(G, B) \tilde{\subseteq}(F, A)$. This relation is denoted by $(F, A)=(G, B)$.

Definition 2.9 [6]. Let $\tau$ be the collection of soft sets over $X$. Then $\tau$ is said to be a soft topology on $X$ if

1) $\tilde{\varnothing}, \tilde{X} \in \tau$;

2) the intersection of any two soft sets in $\tau$ belongs to $\tau$;

3) the union of any number of soft sets in $\tau$ belongs to $\tau$.

The triple $(X, \tau, E)$ is called a soft topological space over $X$. The members of $\tau$ are said to be $\tau$ - soft open sets or soft open sets in $X$. A soft set over $X$ is said to be soft closed in $X$ if its complement belongs to $\tau$. The set of all soft open sets over $X$ denoted by $O S(X, \tau, E)$ or $O S(X)$ and the set of all soft closed sets denoted by $C S(X, \tau, E)$ or $C S(X)$.

Definition $2.10[6]$. The difference of two soft sets $(F, A)$ and $(G, A)$ is defined by $(F, A)-(G, A)=(F-G, A)$, where $(F-G)(e)=F(e)-G(e)$, for all $e \in A$.

Definition 2.11 [6]. Let $(X, \tau, E)$ be a soft topological space and $(F, E) \in S S(X)_{E}$. The soft closure of $(F, E)$, denoted by $\operatorname{cl}(F, E)$ is the intersection of all closed soft super sets of $(F, E)$.

Definition 2.12 [7]. Let $(X, \tau, E)$ be a soft topological space and $(F, E) \in S S(X)_{E}$. The soft interior of $(F, E)$, denoted by $\operatorname{int}(F, E)$ is the union of all open soft subsets of $(F, E)$.

\section{Some Properties of Some Mixed Soft Operations}

In this section we investigated some properties of some mixed operations such as $\left(\tau_{1}, \tau_{2}\right)$-semi open soft, $\left(\tau_{1}, \tau_{2}\right)$-pre open soft. Also we will write $i n t_{1}, c l_{1}, i n t_{2}, c l_{2}$ for $i n t_{\tau_{1}}, c l_{\tau_{1}}, i n t_{\tau_{2}}, c l_{\tau_{2}}$, respectively.

Definition 3.1. Let $X$ be an initial universe and $E$ be a set of parameters. Let $\tau_{1}$ and $\tau_{2}$ be two soft topologies on $X$. Then $(F, E) \in S S(X)_{E}$ is said to be

1) $\left(\tau_{1}, \tau_{2}\right)$-semi open soft if $(F, E) \simeq \tilde{C l}_{2}\left(\right.$ int $\left._{1}(F, E)\right)$;

2) $\left(\tau_{1}, \tau_{2}\right)$-pre open soft if $(F, E) \tilde{\subseteq} \operatorname{int}_{1}\left(c l_{2}(F, E)\right)$;

3) $\left(\tau_{1}, \tau_{2}\right)-\alpha$-open soft if $(F, E) \tilde{\subseteq} \operatorname{int}_{1}\left(c_{2}\left(\operatorname{int}_{1}(F, E)\right)\right)$;

4) $\left(\tau_{1}, \tau_{2}\right)$ - $\beta$-open soft if $(F, E) \subseteq \tilde{c l}_{2}\left(\operatorname{int}_{1}\left(c l_{2}(F, E)\right)\right)$.

The complement of $\left(\tau_{1}, \tau_{2}\right)$-semi open (resp. $\left(\tau_{1}, \tau_{2}\right)$-pre open, $\left(\tau_{1}, \tau_{2}\right)$ - $\alpha$-open, $\left(\tau_{1}, \tau_{2}\right)$ - $\beta$-open) soft set is called $\left(\tau_{1}, \tau_{2}\right)$-semi closed (resp. $\left(\tau_{1}, \tau_{2}\right)$-pre closed, $\left(\tau_{1}, \tau_{2}\right)$ - $\alpha$-closed, $\left(\tau_{1}, \tau_{2}\right)$ - $\beta$-closed) soft (See Figure 1).

Definition 3.2. Let $X$ be an initial universe and $E$ be a set of parameters. Let $\tau_{1}$ and $\tau_{2}$ be two soft topologies on $X$. Then $(F, E) \in S S(X)_{E}$ is said to be

1) $\left(\tau_{1}, \tau_{2}\right)^{\prime}$-semi open soft if $(F, E) \tilde{\subseteq} c l_{1}\left(\right.$ int $\left._{2}(F, E)\right)$;

2) $\left(\tau_{1}, \tau_{2}\right)^{\prime}$-pre open soft if $(F, E) \tilde{\subseteq} \operatorname{int}_{2}\left(c l_{1}(F, E)\right)$;

3) $\left(\tau_{1}, \tau_{2}\right)^{\prime}-\alpha$-open soft if $(F, E) \tilde{\subseteq} \operatorname{int}_{2}\left(c l_{1}\left(\operatorname{int}_{2}(F, E)\right)\right)$;

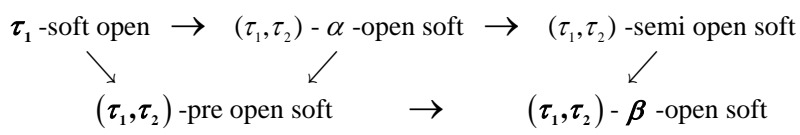

Figure 1. The relations among $\tau_{1}$-soft open, $\left(\tau_{1}, \tau_{2}\right)$ - $\alpha$-open soft, $\left(\tau_{1}, \tau_{2}\right)$-semi open soft, $\left(\tau_{1}, \tau_{2}\right)$-pre open soft and $\left(\tau_{1}, \tau_{2}\right)-\beta$-open soft. 
4) $\left(\tau_{1}, \tau_{2}\right)^{\prime}-\beta$-open soft if $(F, E) \simeq c l_{1}\left(\operatorname{int}_{2}\left(c l_{1}(F, E)\right)\right)$.

The complement of $\left(\tau_{1}, \tau_{2}\right)^{\prime}$-semi open (resp. $\left(\tau_{1}, \tau_{2}\right)^{\prime}$-pre open, $\left(\tau_{1}, \tau_{2}\right)^{\prime}-\alpha$-open, $\left(\tau_{1}, \tau_{2}\right)^{\prime}-\beta$-open) soft set is called $\left(\tau_{1}, \tau_{2}\right)^{\prime}$-semi closed (resp. $\left(\tau_{1}, \tau_{2}\right)^{\prime}$-pre closed, $\left(\tau_{1}, \tau_{2}\right)^{\prime}-\alpha$-closed, $\left(\tau_{1}, \tau_{2}\right)^{\prime}-\beta$-closed) soft (See Figure 2).

Theorem 3.1. Let $X$ be an initial universe and $E$ be a set of parameters. Let $\tau_{1}$ and $\tau_{2}$ be two soft topologies on $X$. Then

1) If $(F, E) \in S S(X)_{E}$ and $(G, E) \in \tau_{1},(G, E) \tilde{\cap} c l_{2}(F, E) \tilde{\subseteq} c l_{2}((G, E) \tilde{\cap}(F, E))$.

2) If $(F, E) \in S S(X)_{E}$ and $(G, E) \in \tau_{2},(G, E) \tilde{\cap} c l_{1}(F, E) \tilde{\subseteq} c l_{1}((G, E) \tilde{\cap}(F, E))$.

Proof. It is seen from Definition 2.11 .

Theorem 3.2. Let $X$ be an initial universe and $E$ be a set of parameters. Let $\tau_{1}$ and $\tau_{2}$ be two soft topologies on $X$. Then

1) $(F, E)$ is a $\left(\tau_{1}, \tau_{2}\right)$-semi open soft set if and only if $c l_{2}(F, E)=c l_{2}\left(\operatorname{int}_{1}(F, E)\right)$.

2) $(F, E)$ is a $\left(\tau_{1}, \tau_{2}\right)^{\prime}$-semi open soft set if and only if $c l_{1}(F, E)=c l_{1}\left(\operatorname{int}_{2}(F, E)\right)$.

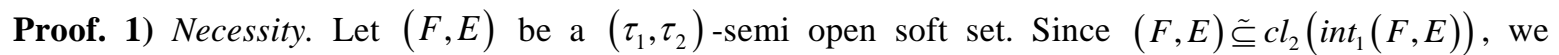
have $c l_{2}(F, E) \tilde{\subseteq} c l_{2}\left(\operatorname{int}_{1}(F, E)\right)$. Also $c l_{2}\left(\operatorname{int}_{1}(F, E)\right) \tilde{\subseteq} c l_{2}(F, E)$. Hence $c l_{2}(F, E)=c l_{2}\left(\operatorname{int}_{1}(F, E)\right)$.

Sufficieny. Let $c l_{2}(F, E)=c l_{2}\left(\operatorname{int}_{1}(F, E)\right)$. Therefore $(F, E) \tilde{\subseteq} l_{2}(F, E)=c l_{2}\left(\operatorname{int}_{1}(F, E)\right)$ and $(F, E)$ is a $\left(\tau_{1}, \tau_{2}\right)$-semi open soft.

2) By a similar way.

Theorem 3.3. Let $X$ be an initial universe and $E$ be a set of parameters. Let $\tau_{1}$ and $\tau_{2}$ be two soft topologies on $X$. Then

1) If $(F, E)$ is a $\tau_{1}$-soft open set and $(G, E)$ is a $\left(\tau_{1}, \tau_{2}\right)$-pre open soft set, $(F, E) \tilde{\cap}(G, E)$ is a $\left(\tau_{1}, \tau_{2}\right)$ pre open soft.

2) If $(F, E)$ is a $\tau_{2}$-soft open set and $(G, E)$ is a $\left(\tau_{1}, \tau_{2}\right)^{\prime}$-pre open soft set, $(F, E) \tilde{\cap}(G, E)$ is a $\left(\tau_{1}, \tau_{2}\right)^{\prime}$-pre open soft.

Proof. (1). Let $(F, E)$ be $\tau_{1}$-soft open and $(G, E)$ be $\left(\tau_{1}, \tau_{2}\right)$-pre open soft set. Then

$$
(F, E) \tilde{\cap}(G, E) \tilde{\subseteq} \operatorname{int}_{1}(F, E) \tilde{\cap} \operatorname{int}_{1}\left(c l_{2}(G, E)\right)=\operatorname{int}_{1}((F, E) \tilde{\cap}(G, E)) \tilde{\subseteq} \operatorname{int}_{1}\left(c l_{2}((F, E) \tilde{\cap}(G, E))\right)
$$

from Theorem 3.1. Hence $(F, E) \tilde{\cap}(G, E)$ is a $\left(\tau_{1}, \tau_{2}\right)$-pre open soft.

(2). By a similar way.

Theorem 3.4. Let $X$ be an initial universe and $E$ be a set of parameters. Let $\tau_{1}$ and $\tau_{2}$ be two soft topologies on $X$. Then

1) If either $(F, E)$ is a $\left(\tau_{1}, \tau_{2}\right)$-semi open soft or $(G, E)$ is a $\left(\tau_{1}, \tau_{2}\right)$-semi open soft set, $\operatorname{int}_{1}\left(c l_{2}((F, E) \tilde{\cap}(G, E))\right)=\operatorname{int}_{1}\left(c l_{2}(F, E)\right) \tilde{\cap} \operatorname{int}_{1}\left(c l_{2}(G, E)\right)$.

2) If either $(F, E)$ is a $\left(\tau_{1}, \tau_{2}\right)^{\prime}$-semi open soft or $(G, E)$ is a $\left(\tau_{1}, \tau_{2}\right)^{\prime}$-semi open soft set, $\operatorname{int}_{2}\left(c_{1}((F, E) \tilde{\cap}(G, E))\right)=\operatorname{int}_{2}\left(c l_{1}(F, E)\right) \tilde{\cap} \operatorname{int}_{2}\left(c l_{1}(G, E)\right)$.

Proof. 1) Let $(F, E),(G, E) \in S S(X)_{E}$. We have

$$
\begin{gathered}
\operatorname{int}_{1}\left(c l_{2}((F, E) \tilde{\cap}(G, E))\right) \tilde{\subseteq} \operatorname{int}_{1}\left(c l_{2}(F, E)\right) \tilde{\cap} \operatorname{int}_{1}\left(c l_{2}(G, E)\right) . \\
\tau_{2} \text {-soft open } \rightarrow\left(\tau_{1}, \tau_{2}\right)^{\prime}-\alpha \text {-open soft } \rightarrow\left(\tau_{1}, \tau_{2}\right)^{\prime} \text {-semi open soft } \\
\searrow \\
\left(\tau_{1}, \tau_{2}\right)^{\prime} \text {-pre open soft }
\end{gathered}
$$

Figure 2. The relations among $\tau_{1}$-soft open, $\left(\tau_{1}, \tau_{2}\right)^{\prime}-\alpha$-open soft, $\left(\tau_{1}, \tau_{2}\right)^{\prime}$-semi open soft, $\left(\tau_{1}, \tau_{2}\right)^{\prime}$-pre open soft and $\left(\tau_{1}, \tau_{2}\right)^{\prime}-\beta$-open soft. 
We assume that $(F, E)$ is a $\left(\tau_{1}, \tau_{2}\right)$-semi open soft set. Then $c l_{2}(F, E)=c l_{2}\left(\operatorname{int}_{1}(F, E)\right)$ from Theorem 3.2. So

$$
\begin{aligned}
& \operatorname{int}_{1}\left(c l_{2}(F, E)\right) \tilde{\cap} \operatorname{int}_{1}\left(c l_{2}(G, E)\right)=\operatorname{int}_{1}\left[c l_{2}(F, E) \tilde{\cap} \operatorname{int}_{1}\left(c l_{2}(G, E)\right)\right] \\
& =\operatorname{int}_{1}\left[c l_{2}\left(\operatorname{int}_{1}(F, E)\right) \tilde{\cap} \operatorname{int}_{1}\left(c l_{2}(G, E)\right)\right] \tilde{\subseteq} \operatorname{int}_{1}\left(c l_{2}\left[\operatorname{int}_{1}(F, E) \tilde{\cap i n t}_{1}\left(c l_{2}(G, E)\right)\right]\right) \\
& =\operatorname{int}_{1}\left(c_{2}\left(\operatorname{int}_{1}\left[\operatorname{int}_{1}(F, E) \tilde{\cap} c_{2}(G, E)\right]\right)\right) \tilde{\subseteq} \operatorname{int}_{1}\left(\operatorname{cl}_{2}\left(\operatorname{int}_{1}\left(c l_{2}\left[\operatorname{int}_{1}(F, E) \tilde{\cap}(G, E)\right]\right)\right)\right) \\
& \tilde{\subseteq} \operatorname{int}_{1}\left(\operatorname{cl}_{2}\left(\operatorname{int}_{1}\left(c l_{2}[(F, E) \tilde{\cap}(G, E)]\right)\right)\right)=\operatorname{int}_{1}\left(c l_{2}[(F, E) \tilde{\subseteq}(G, E)]\right)
\end{aligned}
$$

from Theorem 3.1. Hence we have $\operatorname{int}_{1}\left(c l_{2}((F, E) \tilde{\cap}(G, E))\right)=\operatorname{int}_{1}\left(c l_{2}(F, E)\right) \tilde{\cap} \operatorname{int}_{1}\left(c l_{2}(G, E)\right)$.

2) By a similar way.

Theorem 3.5. Let $X$ be an initial universe and $E$ be a set of parameters. Let $\tau_{1}$ and $\tau_{2}$ be two soft topologies on $X$. Then

1) If $(F, E)$ is a $\tau_{1}$-soft open set and $(G, E)$ is a $\left(\tau_{1}, \tau_{2}\right)$-semi open soft set, $(F, E) \tilde{\cap}(G, E)$ is a $\left(\tau_{1}, \tau_{2}\right)$-semi open soft.

2) If $(F, E)$ is a $\tau_{2}$-soft open set and $(G, E)$ is a $\left(\tau_{1}, \tau_{2}\right)^{\prime}$-semi open soft set, $(F, E) \tilde{\cap}(G, E)$ is a $\left(\tau_{1}, \tau_{2}\right)^{\prime}$-semi open soft.

Proof. 1) Let $(F, E)$ be $\tau_{1}$-open soft and $(G, E)$ be $\left(\tau_{1}, \tau_{2}\right)$-semi open soft set. Then

$$
(F, E) \tilde{\cap}(G, E) \tilde{\subseteq} \operatorname{int}_{1}(F, E) \tilde{\cap} c l_{2}\left(\operatorname{int}_{1}(G, E)\right) \tilde{\subseteq} c l_{2}\left(\operatorname{int}_{1}(F, E) \tilde{\cap} \operatorname{int}_{1}(G, E)\right)=c l_{2}\left(\operatorname{int}_{1}((F, E) \tilde{\cap}(G, E))\right)
$$

from Theorem 3.1. Therefore $(F, E) \tilde{\cap}(G, E)$ is a $\left(\tau_{1}, \tau_{2}\right)$-semi open soft.

2) By a similar way.

Theorem 3.6. Let $X$ be an initial universe and $E$ be a set of parameters. Let $\tau_{1}$ and $\tau_{2}$ be two soft topologies on $X$. Let $(F, E),(G, E) \in S S(X)_{E}$, then

1) $(F, E)$ is $\left(\tau_{1}, \tau_{2}\right)-\alpha$-open soft set if and only if there exists a $(G, E) \tau_{1}$-soft open set such that $(G, E) \tilde{\subseteq}(F, E) \tilde{\subseteq} \operatorname{int}_{1}\left(c l_{2}(G, E)\right)$.

2) If $(F, E)$ is $\left(\tau_{1}, \tau_{2}\right)$ - $\alpha$-open soft set and $(F, E) \tilde{\subseteq}(G, E) \tilde{\subseteq} \operatorname{int}_{1}\left(c l_{2}(F, E)\right)$, then $(G, E)$ is $\left(\tau_{1}, \tau_{2}\right)$ $\alpha$-open soft.

3) $(F, E)$ is $\left(\tau_{1}, \tau_{2}\right)^{\prime}-\alpha$-open soft set if and only if there exists a $(G, E) \tau_{2}$-soft open set such that $(G, E) \tilde{\subseteq}(F, E) \tilde{\subseteq} \operatorname{int}_{2}\left(c l_{1}(G, E)\right)$.

4) If $(F, E)$ is $\left(\tau_{1}, \tau_{2}\right)^{\prime}-\alpha$-open soft set and $(F, E) \tilde{\subseteq}(G, E) \tilde{\subseteq} \operatorname{int}_{2}\left(c l_{1}(F, E)\right)$, then $(G, E)$ is $\left(\tau_{1}, \tau_{2}\right)^{\prime}$ $\alpha$-open soft.

Proof. 1) Necessity. Let $\operatorname{int}_{1}(F, E)=(G, E)$. Then

$$
(G, E) \tilde{\subseteq}(F, E) \tilde{\subseteq} \operatorname{int}_{1}\left(c l_{2}\left(\operatorname{int}_{1}(F, E)\right)\right)=\operatorname{int}_{1}\left(c l_{2}(F, E)\right) .
$$

Sufficiency. Let $(G, E)$ is $\tau_{1}$-soft open set and $(G, E) \tilde{\subseteq}(F, E) \tilde{\subseteq i n t}\left(c_{2}(G, E)\right)$. Then $\operatorname{int}_{1}(G, E)=(G, E) \tilde{\subseteq} \operatorname{int}_{1}(F, E)$. Hence $(F, E) \tilde{\subseteq} \operatorname{int}_{1}\left(c_{2}\left(\operatorname{int}_{1}(G, E)\right)\right) \tilde{\subseteq} \operatorname{int}_{1}\left(\operatorname{cl}_{2}\left(\operatorname{int}_{1}(F, E)\right)\right)$. Thus $(F, E)$ is $\left(\tau_{1}, \tau_{2}\right)-\alpha$-open soft.

2) Let $(F, E)$ is $\left(\tau_{1}, \tau_{2}\right)-\alpha$-open soft set and $(F, E) \tilde{\subseteq}(G, E) \tilde{\subseteq} i n t_{1}\left(c l_{2}(F, E)\right)$. Hence

$$
(F, E) \tilde{\subseteq}(G, E) \tilde{\subseteq} \operatorname{int}_{1}\left(\operatorname{cl}_{2}\left(\operatorname{int}_{1}\left(\operatorname{cl}_{2}\left(\operatorname{int}_{1}(F, E)\right)\right)\right)\right) \tilde{\subseteq} \operatorname{int}_{1}\left(\operatorname{cl}_{2}\left(\operatorname{int}_{1}(F, E)\right)\right) \tilde{\subseteq} \operatorname{int}_{1}\left(\operatorname{cl}_{2}\left(\operatorname{int}_{1}(G, E)\right)\right) .
$$

Thus $(G, E)$ is $\left(\tau_{1}, \tau_{2}\right)-\alpha$-open soft.

3)-4) By a similar way.

Theorem 3.7. Let $X$ be an initial universe and $E$ be a set of parameters. Let $\tau_{1}$ and $\tau_{2}$ be two soft topologies on $X$. Then 
1) If $(F, E)$ is $\left(\tau_{1}, \tau_{2}\right)-\alpha$-open soft and $(G, E)$ is $\left(\tau_{1}, \tau_{2}\right)-\beta$-open soft, then $(F, E) \tilde{\bigcap}(G, E)$ is $\left(\tau_{1}, \tau_{2}\right)$ - $\beta$-open soft.

2) If $(F, E)$ is $\left(\tau_{1}, \tau_{2}\right)^{\prime}-\alpha$-open soft and $(G, E)$ is $\left(\tau_{1}, \tau_{2}\right)^{\prime}-\beta$-open soft, then $(F, E) \tilde{\bigcap}(G, E)$ is $\left(\tau_{1}, \tau_{2}\right)^{\prime}-\beta$-open soft.

Proof. 1) Let $(F, E)$ is $\left(\tau_{1}, \tau_{2}\right)$ - $\alpha$-open soft and $(G, E)$ is $\left(\tau_{1}, \tau_{2}\right)-\beta$-open soft. Then

$$
\begin{aligned}
& (F, E) \tilde{\subseteq}(G, E) \tilde{\subseteq} \operatorname{int}_{1}\left(c l_{2}\left(\operatorname{int}_{1}(F, E)\right)\right) \tilde{\subseteq} l_{2}\left(\operatorname{int}_{1}\left(c l_{2}(G, E)\right)\right) \\
& \tilde{\subseteq} c l_{2}\left[\operatorname{int}_{1}\left(c l_{2}\left(\operatorname{int}_{1}(F, E)\right)\right) \tilde{\cap} \operatorname{int}_{1}\left(c l_{2}(G, E)\right)\right]=c l_{2}\left(\operatorname{int}_{1}\left[c l_{2}\left(\operatorname{int}_{1}(F, E)\right) \tilde{\cap} \operatorname{int}_{1}\left(c l_{2}(G, E)\right)\right]\right) \\
& \tilde{\subseteq} c_{2}\left(\operatorname{int}_{1}\left(c l_{2}\left[\operatorname{int}_{1}(F, E) \tilde{\cap} \operatorname{int}_{1}\left(c l_{2}(G, E)\right)\right]\right)\right)=c l_{2}\left(\operatorname{int}_{1}\left(\operatorname{cl}_{2}\left(\operatorname{int}_{1}\left[\operatorname{int}_{1}(F, E) \tilde{\cap} c_{2}(G, E)\right]\right)\right)\right) \\
& \tilde{\subseteq} c_{2}\left(\operatorname{int}_{1}\left(\operatorname{cl}_{2}\left(\operatorname{int}_{1}\left(c l_{2}\left[\operatorname{int}_{1}(F, E) \tilde{\cap}(G, E)\right]\right)\right)\right)\right) \tilde{\subseteq} c_{2}\left(\operatorname{int}_{1}\left(c l_{2}\left(\operatorname{int}_{1}\left(c l_{2}[(F, E) \tilde{\cap}(G, E)]\right)\right)\right)\right) \\
& \tilde{\subseteq} c_{2}\left(\operatorname{int}_{1}\left(c l_{2}[(F, E) \tilde{\cap}(G, E)]\right)\right)
\end{aligned}
$$

from Theorem 3.1. Thus $(F, E) \tilde{\subseteq}(G, E)$ is $\left(\tau_{1}, \tau_{2}\right)$ - $\beta$-open soft.

2) By a similar way.

Proposition 3.1. Let $X$ be an initial universe and $E$ be a set of parameters. Let $\tau_{1}$ and $\tau_{2}$ be two soft topologies on $X$. If $(F, E) \in S S(X)_{E}$, the following statements hold:

1) $\operatorname{int}_{1}\left(c l_{2}(F, E)^{c}\right)=\tilde{X}-c l_{1}\left(\operatorname{int}_{2}(F, E)\right)$.

2) $\operatorname{cl}_{2}\left(\operatorname{int}_{1}(F, E)^{c}\right)=\tilde{X}-\operatorname{int}_{2}\left(c l_{1}(F, E)\right)$.

Proof. It is obvious from Definition 2.1., 2.11. and 2.12.

Theorem 3.8. Let $X$ be an initial universe and $E$ be a set of parameters. Let $\tau_{1}$ and $\tau_{2}$ be two soft topologies on $X$. Then

1) $(F, E) \in S S(X)_{E}$ is a $\left(\tau_{1}, \tau_{2}\right)$-pre closed soft set if and only if $c l_{1}\left(\operatorname{int}_{2}(F, E)\right) \tilde{\subseteq}(F, E)$.

2) $(F, E) \in S S(X)_{E}$ is a $\left(\tau_{1}, \tau_{2}\right)^{\prime}$-pre closed soft set if and only if $\operatorname{cl}_{2}\left(\operatorname{int}_{1}(F, E)\right) \tilde{\subseteq}(F, E)$.

Proof. 1) Necessity. Let $(F, E)$ be $\left(\tau_{1}, \tau_{2}\right)$-pre closed soft set. Then $(F, E)^{c}$ is a $\left(\tau_{1}, \tau_{2}\right)$-pre open soft set, that is

$$
(F, E)^{c} \tilde{\subseteq} \operatorname{int}_{1}\left(c l_{2}(F, E)^{c}\right)=\operatorname{int}_{1}\left(c l_{2}(\tilde{X}-(F, E))\right)=\tilde{X}-\left(c l_{1}\left(\operatorname{int}_{2}(F, E)\right)\right)
$$

Thus $\operatorname{cl}_{1}\left(\operatorname{int}_{2}(F, E)\right) \tilde{\subseteq}(F, E)$.

Sufficiency. Let $c l_{1}\left(\operatorname{int}_{2}(F, E)\right) \tilde{\subseteq}(F, E)$, then $\tilde{X}-(F, E) \tilde{\subseteq} \tilde{X}-c l_{1}\left(\operatorname{int}_{2}(F, E)\right)=\operatorname{int}_{1}\left(c l_{2}(\tilde{X}-(F, E))\right)$. Hence $\tilde{X}-(F, E)$ is a $\left(\tau_{1}, \tau_{2}\right)$-pre open soft set. Therefore $(F, E)$ is a $\left(\tau_{1}, \tau_{2}\right)$-pre closed soft set.

2) By a similar way.

Theorem 3.9. Let $X$ be an initial universe and $E$ be a set of parameters. Let $\tau_{1}$ and $\tau_{2}$ be two soft topologies on $X$. Then

1) $(F, E) \in S S(X)_{E}$ is a $\left(\tau_{1}, \tau_{2}\right)-\alpha$-closed soft set if and only if $c l_{1}\left(\operatorname{int}_{2}\left(c l_{1}(F, E)\right)\right) \tilde{\subseteq}(F, E)$.

2) $(F, E) \in S S(X)_{E}$ is a $\left(\tau_{1}, \tau_{2}\right)^{\prime}-\alpha$-closed soft set if and only if $\operatorname{cl}_{2}\left(\operatorname{int}_{1}\left(c_{2}(F, E)\right)\right) \tilde{\subseteq}(F, E)$.

Proof. 1) Necessity. Let $(F, E)$ is a $\left(\tau_{1}, \tau_{2}\right)$ - $\alpha$-closed soft set. Then $(F, E)^{c}$ is a $\left(\tau_{1}, \tau_{2}\right)$ - $\alpha$-open soft, that is $(F, E)^{c} \tilde{\subseteq} \operatorname{int}_{1}\left(c l_{2}\left(\operatorname{int}_{1}(F, E)^{c}\right)\right)=\operatorname{int}_{1}\left(c_{2}\left(\operatorname{int}_{1}(\tilde{X}-(F, E))\right)\right)=\tilde{X}-c l_{1}\left(\operatorname{int}_{2}\left(c l_{1}(F, E)\right)\right)$. Therefore, $c l_{1}\left(\operatorname{int}_{2}\left(c l_{1}(F, E)\right)\right) \tilde{\subseteq}(F, E)$.

Sufficiency. Let $c l_{1}\left(\operatorname{int}_{2}\left(c l_{1}(F, E)\right)\right) \tilde{\subseteq}(F, E)$, then

$$
\tilde{X}-(F, E) \tilde{\subseteq} \tilde{X}-c l_{1}\left(\operatorname{int}_{2}\left(c l_{1}(F, E)\right)\right)=\operatorname{int}_{1}\left(c l_{2}\left(\operatorname{int}_{1}(F, E)\right)\right) .
$$

Hence $\tilde{X}-(F, E)$ is a $\left(\tau_{1}, \tau_{2}\right)-\alpha$-open soft set. Therefore $(F, E)$ is a $\left(\tau_{1}, \tau_{2}\right)-\alpha$-closed soft set. 
2) By a similar way.

Theorem 3.10. Let $X$ be an initial universe and $E$ be a set of parameters. Let $\tau_{1}$ and $\tau_{2}$ be two soft topologies on $X$. Then

1) $(F, E) \in S S(X)_{E}$ is a $\left(\tau_{1}, \tau_{2}\right)$-semi closed soft set if and only if $\operatorname{int}_{2}\left(c l_{1}(F, E)\right) \tilde{\subseteq}(F, E)$.

2) $(F, E) \in S S(X)_{E}$ is a $\left(\tau_{1}, \tau_{2}\right)^{\prime}$-semi closed soft set if and only if $\operatorname{int}_{1}\left(c_{2}(F, E)\right) \tilde{\subseteq}(F, E)$.

Proof. 1) Necessity. Let $(F, E)$ is a $\left(\tau_{1}, \tau_{2}\right)$-semi closed soft set. Then $(F, E)^{c}$ is a $\left(\tau_{1}, \tau_{2}\right)$-semi open soft set, that is $(F, E)^{c} \tilde{\subseteq} c l_{2}\left(\operatorname{int}_{1}(F, E)^{c}\right)=c_{2}\left(\operatorname{int}_{1}(\tilde{X}-(F, E))\right)=\tilde{X}-\operatorname{int}_{2}\left(c l_{1}(F, E)\right)$. Therefore, $\operatorname{int}_{2}\left(c l_{1}(F, E)\right) \tilde{\subseteq}(F, E)$.

Sufficiency. Let $\operatorname{int}_{2}\left(c l_{1}(F, E)\right) \tilde{\subseteq}(F, E)$, then $\tilde{X}-(F, E) \tilde{\subseteq} \tilde{X}-\operatorname{int}_{2}\left(c l_{1}(F, E)\right)=\operatorname{cl}_{2}\left(\operatorname{int}_{1}(\tilde{X}-(F, E))\right)$. Hence $\tilde{X}-(F, E)$ is a $\left(\tau_{1}, \tau_{2}\right)$-semi open soft set. Therefore $(F, E)$ is a $\left(\tau_{1}, \tau_{2}\right)$-semi closed soft set.

2) By a similar way.

Theorem 3.11. Let $X$ be an initial universe and $E$ be a set of parameters. Let $\tau_{1}$ and $\tau_{2}$ be two soft topologies on $X$. Then

1) If $(F, E)$ is a $\left(\tau_{1}, \tau_{2}\right)-\alpha$-open soft set and $(G, E)$ is a $\left(\tau_{1}, \tau_{2}\right)$-semi open soft set, $(F, E) \tilde{\cap}(G, E)$ is a $\left(\tau_{1}, \tau_{2}\right)$-semi open soft.

2) If $(F, E)$ is a $\left(\tau_{1}, \tau_{2}\right)^{\prime}-\alpha$-open soft set and $(G, E)$ is a $\left(\tau_{1}, \tau_{2}\right)^{\prime}$-semi open soft set, $(F, E) \tilde{\cap}(G, E)$ is a $\left(\tau_{1}, \tau_{2}\right)^{\prime}$-semi open soft.

Proof. 1) Let $(F, E)$ is a $\left(\tau_{1}, \tau_{2}\right)-\alpha$-open soft and $(G, E)$ is a $\left(\tau_{1}, \tau_{2}\right)$-semi open soft. Then

$$
\begin{aligned}
& (F, E) \tilde{\cap}(G, E) \\
& \tilde{\subseteq} \operatorname{int}_{1}\left(c l_{2}\left(\operatorname{int}_{1}(F, E)\right)\right) \tilde{\cap} c l_{2}\left(\operatorname{int}_{1}(G, E)\right) \tilde{\subseteq} c l_{2}\left[\operatorname{int}_{1}\left(c l_{2}\left(\operatorname{int}_{1}(F, E)\right)\right) \tilde{\cap} \operatorname{int}_{1}(G, E)\right] \\
& \tilde{\subseteq} c_{2}\left(\operatorname{int}_{1}\left[c l_{2}\left(\operatorname{int}_{1}(F, E)\right) \tilde{\cap} \operatorname{int}_{1}(G, E)\right]\right) \tilde{\subseteq} c l_{2}\left(\operatorname{int}_{1}\left(c l_{2}\left[\operatorname{int}_{1}(F, E) \tilde{\cap} \operatorname{int}_{1}(G, E)\right]\right)\right) \\
& \tilde{\subseteq} c_{2}\left(\operatorname{int}_{1}\left(c l_{2}\left(\operatorname{int}_{1}[(F, E) \tilde{\cap}(G, E)]\right)\right)\right) \tilde{\subseteq} c_{2}\left(\operatorname{int}_{1}((F, E) \tilde{\cap}(G, E))\right)
\end{aligned}
$$

Therefore $(F, E) \tilde{\cap}(G, E)$ is a $\left(\tau_{1}, \tau_{2}\right)$-semi open soft.

2) By a similar way.

Theorem 3.12. Let $X$ be an initial universe and $E$ be a set of parameters. Let $\tau_{1}$ and $\tau_{2}$ be two soft topologies on $X$. Then

1) $(F, E) \in S S(X)_{E}$ is a $\left(\tau_{1}, \tau_{2}\right)-\beta$-closed soft set if and only if $\operatorname{int}_{2}\left(\operatorname{cl}_{1}\left(\operatorname{int}_{2}(F, E)\right)\right) \tilde{\subseteq}(F, E)$.

2) $(F, E) \in S S(X)_{E}$ is a $\left(\tau_{1}, \tau_{2}\right)^{\prime}-\beta$-closed soft set if and only if $\operatorname{int}_{1}\left(\operatorname{cl}_{2}\left(\operatorname{int}_{1}(F, E)\right)\right) \tilde{\subseteq}(F, E)$.

Proof. 1) Necessity. Let $(F, E)$ is a $\left(\tau_{1}, \tau_{2}\right)$ - $\beta$-closed set. Then $(F, E)^{c}$ is a $\left(\tau_{1}, \tau_{2}\right)$ - $\beta$-open soft set, that is $(F, E)^{c} \tilde{\subseteq} c l_{2}\left(\operatorname{int}_{1}\left(c_{2}(F, E)^{c}\right)\right)=c l_{2}\left(\operatorname{int}_{1}\left(c l_{2}(\tilde{X}-(F, E))\right)\right)=\tilde{X}-\operatorname{int}_{2}\left(c l_{1}\left(\operatorname{int}_{2}(F, E)\right)\right)$. Therefore, $\operatorname{int}_{2}\left(c l_{1}\left(\operatorname{int}_{2}(F, E)\right)\right) \tilde{\subseteq}(F, E)$.

Sufficiency. Let $\operatorname{int}_{2}\left(c l_{1}\left(\operatorname{int}_{2}(F, E)\right)\right) \tilde{\subseteq}(F, E)$. Then

$$
\tilde{X}-(F, E) \tilde{\subseteq} \tilde{X}-\operatorname{int}_{2}\left(c_{1}\left(\operatorname{int}_{2}(F ; E)\right)\right)=\operatorname{cl}_{2}\left(\operatorname{int}_{1}\left(c l_{2}(\tilde{X}-(F, E))\right)\right) .
$$

Hence $(F, e)^{c}$ is a $\left(\tau_{1}, \tau_{2}\right)$ - $\beta$-open soft set. Therefore, $(F, E)$ is a $\left(\tau_{1}, \tau_{2}\right)$ - $\beta$-closed soft.

2) By a similar way.

\section{Extremally Soft Disconnectedness on Two Soft Topologies}

In this section we introduced extremally soft disconnectedness (briefly, ESDC) via two soft topological spaces over $X$ and investigated some characterizations of ESDC.

Definition 4.1. Let $X$ be an initial universe and $E$ be a set of parameters. Let $\tau_{1}$ and $\tau_{2}$ be two soft 
topologies on $X . \tau_{1}$ is said to be $\tau_{2}$-ESDC if $(F, E) \in \tau_{1}$ implies that $c_{2}(F, E) \in \tau_{1}$.

Definition 4.2. Let $X$ be an initial universe and $E$ be a set of parameters. Let $\tau_{1}$ and $\tau_{2}$ be two soft topologies on $X .(F, E)$ is said to be $\left(\tau_{1}, \tau_{2}\right)$-regular open soft set if $(F, E)=\operatorname{int}_{1}\left(c l_{2}(F, E)\right)$.

Theorem 4.1. Let $X$ be an initial universe and $E$ be a set of parameters. Let $\tau_{1}$ and $\tau_{2}$ be two soft topologies on $X$. Then the following statements are equivalent:

1) $\tau_{1}$ is $\tau_{2}$-ESDC.

2) If $(F, E)=c l_{1}(F, E) \operatorname{int}_{2}(F, E)=c l_{1}\left(\operatorname{int}_{2}(F, E)\right)$.

3) If $(F, E) \in S S(X)_{E}, \quad c l_{2}\left(\operatorname{int}_{1}(F, E)\right) \tilde{\subseteq} \operatorname{int}_{1}\left(c l_{2}(F, E)\right)$.

4) Every $\left(\tau_{1}, \tau_{2}\right)$-semi open soft set is $\left(\tau_{1}, \tau_{2}\right)$-pre open soft.

5) If $(F, E)$ is a $\left(\tau_{1}, \tau_{2}\right)$ - $\beta$-open soft set, $c l_{2}(F, E) \in \tau_{1}$.

6) Every $\left(\tau_{1}, \tau_{2}\right)$ - $\beta$-open soft set is $\left(\tau_{1}, \tau_{2}\right)$-pre open soft.

7) Every $\left(\tau_{1}, \tau_{2}\right)$-semi open soft set is $\left(\tau_{1}, \tau_{2}\right)$ - $\alpha$-open soft.

8) If $(F, E)$ is a $\left(\tau_{1}, \tau_{2}\right)$-regular open soft set, $(F, E)=c l_{2}(F, E)$.

Proof. 1) $\Rightarrow$ 2) Let $(F, E)=c l_{1}(F, E)$. Then $\tilde{X}-(F, E)=i n t_{1}(\tilde{X}-(F, E))$, that is $(F, E)^{c} \in \tau_{1}$. From 1), $c_{2}(F, E)^{c} \in \tau_{1}$. Hence int ${ }_{1}\left(c l_{2}(F, E)^{c}\right)=c l_{2}(F, E)^{c}$. Therefore, $\operatorname{int}_{2}(F, E)=c l_{1}\left(\operatorname{int}_{2}(F, E)\right)$.

2) $\Rightarrow 3)$ Let $(F, E) \in S S(X)_{E}$, Then $\tilde{X}-\operatorname{int}_{1}(F, E)$ is $\tau_{1}$-soft closed and from 2) $\operatorname{int}_{2}\left(\tilde{X}-\operatorname{int}_{1}(F, E)\right)$ is $\tau_{1}$-soft closed. Therefore, $\operatorname{cl}_{2}\left(\operatorname{int}_{1}(F, E)\right)=\tilde{X}-\operatorname{int}_{2}\left(\tilde{X}-i n t_{1}(F, E)\right)$ is $\tau_{1}$-soft open and since $c l_{2}\left(\operatorname{int}_{1}(F, E)\right) \tilde{\subseteq} c l_{2}(F, E)$, we have $c l_{2}\left(\operatorname{int}_{1}(F, E)\right) \tilde{\subseteq} i n t_{1}\left(c l_{2}(F, E)\right)$.

$3) \Rightarrow 4)$ Let $(F, E)$ is a $\left(\tau_{1}, \tau_{2}\right)$-semi open soft set. Then $(F, E) \tilde{\subseteq} c_{2}\left(\operatorname{int}_{1}(F, E)\right) \tilde{\subseteq} \operatorname{int}_{1}\left(\operatorname{cl}_{2}(F, E)\right)$ from 3). Hence, $(F, E)$ is a $\left(\tau_{1}, \tau_{2}\right)$-pre open soft.

$4) \Rightarrow 5)$ Let $(F, E)$ is a $\left(\tau_{1}, \tau_{2}\right)-\beta$-open soft set, that is $(F, E) \tilde{\subseteq} \operatorname{cl}_{2}\left(\operatorname{int}_{1}\left(c l_{2}(F, E)\right)\right)$. Then

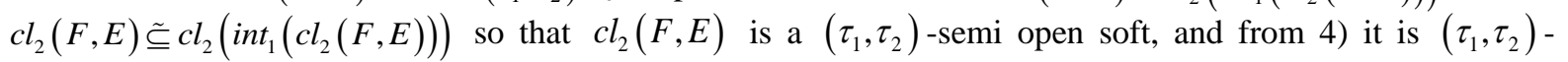
pre open soft. $c l_{2}(F, E) \tilde{\subseteq i n t_{1}}\left(c l_{2}\left(c l_{2}(F, E)\right)\right)=i n t_{1}\left(c l_{2}(F, E)\right)$. Hence, $c l_{2}(F, E) \in \tau_{1}$.

$5) \Rightarrow 6)$ Let $(F, E)$ is a $\left(\tau_{1}, \tau_{2}\right)-\beta$-open soft set. From 5), $c l_{2}(F, E) \in \tau_{1}$ and $c l_{2}(F, E)=\operatorname{int}_{1}\left(c l_{2}(F, E)\right)$.

Hence, $(F, E) \tilde{\subseteq} c l_{2}(F, E)=i n t_{1}\left(c l_{2}(F, E)\right)$. Therefore, $(F, E)$ is a $\left(\tau_{1}, \tau_{2}\right)$-pre open soft.

6) $\Rightarrow 7)$ Let $(F, E)$ is a $\left(\tau_{1}, \tau_{2}\right)$-semi open soft set, then $(F, E)$ is a $\left(\tau_{1}, \tau_{2}\right)$ - $\beta$-open soft. From 6$)$, $(F, E)$ is a $\left(\tau_{1}, \tau_{2}\right)$-pre open soft. Hence, $(F, E)$ is a $\left(\tau_{1}, \tau_{2}\right)-\alpha$-open soft.

$7) \Rightarrow$ 1) Let $(F, E) \in \tau_{1}$. Then, $(F, E)=i n t_{1}(F, E)$ so that

$$
c l_{2}(F, E)=c l_{2}\left(\operatorname{int}_{1}(F, E)\right) \tilde{\subseteq} c l_{2}\left(\operatorname{int}_{1}\left(c l_{2}(F, E)\right)\right)
$$

and $\mathrm{Cl}_{2}(F, E)$ is $\left(\tau_{1}, \tau_{2}\right)$-semi open soft. From 7$), \mathrm{Cl}_{2}(F, E)$ is $\left(\tau_{1}, \tau_{2}\right)-\alpha$-open soft. Hence

$$
c l_{2}(F, E) \tilde{\subseteq} \operatorname{int}_{1}\left(c l_{2}\left(\operatorname{int}_{1}\left(c l_{2}(F, E)\right)\right)\right)=\operatorname{int}_{1}\left(c l_{2}(F, E)\right) \tilde{\subseteq} c l_{2}(F, E)
$$

and $c l_{2}(F, E)=i n t_{1}\left(c l_{2}(F, E)\right)$ so that $c l_{2}(F, E) \in \tau_{1}$.

1) $\Rightarrow 8)$ Let $(F, E)$ is $\left(\tau_{1}, \tau_{2}\right)$-regular open soft. Then $(F, E) \in \tau_{1}$ and $c l_{2}(F, E) \in \tau_{1}$ from 1$)$. Therefore, $\operatorname{cl}_{2}(F, E)=\operatorname{int}_{1}\left(c l_{2}(F, E)\right)=(F, E)$.

8) $\Rightarrow$ 1) Let $(F, E) \in \tau_{1}$. Since $\operatorname{int}_{1}\left(c l_{2}(F, E)\right)=\operatorname{int}_{1}\left(c l_{2}\left(\operatorname{int}_{1}\left(c l_{2}(F, E)\right)\right)\right)$, the soft set $\operatorname{int}_{1}\left(c_{2}(F, E)\right)$ is $\left(\tau_{1}, \tau_{2}\right)$-regular open soft. From 8), $\operatorname{int}_{1}\left(c l_{2}(F, E)\right)=c l_{2}\left(\operatorname{int}_{1}\left(c l_{2}(F, E)\right)\right)$. Since $(F, E) \tilde{\subseteq} l_{2}(F, E)$,

$$
(F, E) \tilde{\subseteq} \operatorname{int}_{1}\left(c l_{2}(F, E)\right)=c l_{2}\left(\operatorname{int}_{1}\left(c l_{2}(F, E)\right)\right) .
$$

Hence, we have $c l_{2}(F, E) \subseteq{ }_{c l}\left(\operatorname{int}_{1}\left(c l_{2}(F, E)\right)\right)=\operatorname{int}_{1}\left(c l_{2}(F, E)\right)$ so that $c l_{2}(F, E) \in \tau_{1}$.

Theorem 4.2. Let $X$ be an initial universe and $E$ be a set of parameters. Let $\tau_{1}$ and $\tau_{2}$ be two soft topologies on $X$. Then the following statements are equivalent:

1) $\tau_{1}$ is $\tau_{2}$-ESDC. 
2) If $(F, E)$ is $\left(\tau_{1}, \tau_{2}\right)$-semi open soft set, $c l_{2}(F, E) \in \tau_{1}$.

3) If $(F, E)$ is $\left(\tau_{1}, \tau_{2}\right)$-pre open soft set, $c l_{2}(F, E) \in \tau_{1}$.

4) If $(F, E)$ is $\left(\tau_{1}, \tau_{2}\right)$-regular open soft set, $c l_{2}(F, E) \in \tau_{1}$.

Proof. 1) $\Rightarrow 2$ ) Every $\left(\tau_{1}, \tau_{2}\right)$-semi open soft set is $\left(\tau_{1}, \tau_{2}\right)$ - $\beta$-open soft so that $\operatorname{cl}_{2}(F, E) \in \tau_{1}$ from Theorem 4.1.

$2) \Rightarrow 4)$. Every $\left(\tau_{1}, \tau_{2}\right)$-regular open soft set is $\left(\tau_{1}, \tau_{2}\right)$-semi open soft set since

$$
(F, E)=\operatorname{int}_{1}\left(c l_{2}(F, E)\right) \tilde{\subseteq} c l_{2}\left(\operatorname{int}_{1}\left(c l_{2}(F, E)\right)\right)=c_{2}\left(\operatorname{int}_{1}\left(\operatorname{int}_{1}\left(c l_{2}(F, E)\right)\right)\right)=c l_{2}\left(\operatorname{int}_{1}(F, E)\right) .
$$

From 2), $\mathrm{cl}_{2}(F, E) \in \tau_{1}$.

1) $\Rightarrow 3$ ) Since every $\left(\tau_{1}, \tau_{2}\right)$-pre open soft set is $\left(\tau_{1}, \tau_{2}\right)$ - $\beta$-open soft, it is obvious from Theorem 4.1.

3) $\Rightarrow 4$ ) Since every $\left(\tau_{1}, \tau_{2}\right)$-regular open soft set is $\left(\tau_{1}, \tau_{2}\right)$-pre open soft, $c l_{2}(F, E) \in \tau_{1}$.

4) $\Rightarrow$ 1) If $(F, E) \in \tau_{1}$, since $\operatorname{int}_{1}\left(c l_{2}(F, E)\right)=\operatorname{int}_{1}\left(c l_{2}\left(\operatorname{int}_{1}\left(c l_{2}(F, E)\right)\right)\right)$ and we have $\operatorname{int}_{1}\left(c l_{2}(F, E)\right)$ is $\left(\tau_{1}, \tau_{2}\right)$-regular open soft. Also we obtain from 4) $c l_{2}\left(\operatorname{int}_{1}\left(c l_{2}(F, E)\right)\right) \in \tau_{1}$ and $(F, E) \tilde{\subseteq} \operatorname{int}_{1}\left(c l_{2}(F, E)\right)$ by $(F, E) \tilde{\subseteq} C l_{2}(F, E)$ so that

$$
\begin{aligned}
& (F, E) \simeq \operatorname{int}_{1}\left(c l_{2}(F, E)\right) \subseteq \operatorname{cl}_{2}\left(\operatorname{int}_{1}\left(c l_{2}(F, E)\right)\right)=\operatorname{int}_{1}\left(c l_{2}\left(\operatorname{int}_{1}\left(c l_{2}(F, E)\right)\right)\right)=\operatorname{int}_{1}\left(c l_{2}(F, E)\right), \\
& c l_{2}(F, E) \tilde{\simeq} c_{2}\left(\operatorname{int}_{1}\left(c l_{2}(F, E)\right)\right)=\operatorname{int}_{1}\left(c l_{2}(F, E)\right) .
\end{aligned}
$$

Therefore $\mathrm{Cl}_{2}(F, E) \in \tau_{1}$.

Lemma 4.1. $\tau_{1} \tilde{\subseteq} \tau_{2}$ implies $\operatorname{int}_{1}(F, E) \tilde{\subseteq} \operatorname{int}_{2}(F, E)$ and $c l_{2}(F, E) \tilde{\subseteq} c l_{1}(F, E)$ for $(F, E) \in S S(X)_{E}$.

Proof. Obvious.

Theorem 4.3. Let $X$ be an initial universe and $E$ be a set of parameters. Let $\tau_{1}, \tau_{2}$ be two soft topologies on $X$ and $\tau_{1} \tilde{\subseteq} \tau_{2}$. Then the following statements are equivalent:

1) $\tau_{1}$ is $\tau_{2}$-ESDC.

2) If $(F, E) \in \tau_{1}$ and $(G, E) \in \tau_{2}, \quad c l_{2}(F, E) \tilde{\cap} c l_{1}(G, E) \tilde{\subseteq} c l_{1}((F, E) \tilde{\cap}(G, E))$.

3) If $(F, E) \in \tau_{1}, \quad(G, E) \in \tau_{2}$ and $(F, E) \tilde{\bigcap}(G, E)=\tilde{\varnothing}, \quad c l_{2}(F, E) \tilde{\cap} c l_{1}(G, E)=\tilde{\varnothing}$.

4) If $(F, E) \in S S(X)_{E},(G, E) \in \tau_{2}$ and $(F, E) \tilde{\cap}(G, E)=\tilde{\varnothing}, \quad c l_{2}\left(\operatorname{int}_{1}\left(c l_{2}(F, E)\right)\right) \tilde{\cap} c l_{1}(G, E)=\tilde{\varnothing}$.

Proof. 1) $\Rightarrow 2)$ Let $(F, E) \in \tau_{1}$ and $(G, E) \in \tau_{2}$. From (1), $c l_{2}(F, E) \in \tau_{2}$. Then

$$
\begin{aligned}
& c l_{2}(F, E) \tilde{\cap} c l_{1}(G, E) \tilde{\subseteq} c l_{1}\left(c l_{2}(F, E) \tilde{\cap}(G, E)\right) \tilde{\subseteq} c l_{1}\left(c l_{2}((F, E) \tilde{\cap}(G, E))\right) \\
& \tilde{\subseteq} c l_{1}\left(c l_{1}((F, E) \tilde{\cap}(G, E))\right)=c l_{1}((F, E) \tilde{\cap}(G, E))
\end{aligned}
$$

2) $\Rightarrow$ 3) Let $(F, E) \in \tau_{1},(G, E) \in \tau_{2}$ and $(F, E) \tilde{\cap}(G, E)=\tilde{\varnothing}$. From (2),

$$
c l_{2}(F, E) \tilde{\cap} c l_{1}(G, E) \tilde{\subseteq} c l_{1}((F, E) \tilde{\cap}(G, E))=\tilde{\varnothing} .
$$

$3) \Rightarrow 4)$ Let $(F, E) \in S S(X)_{E},(G, E) \in \tau_{2}$ and $(F, E) \tilde{\cap}(G, E)=\tilde{\varnothing}$. From (3), $\operatorname{int}_{1}\left(c_{2}(F, E)\right) \in \tau_{1}$, $(G, E) \in \tau_{2}$ and $c l_{2}(F, E) \tilde{\cap}(G, E)=\tilde{\varnothing}$, $\operatorname{int}_{1}\left(c l_{2}(F, E)\right) \tilde{\cap}(G, E)=\tilde{\varnothing}$. Hence

$$
c l_{2}\left(\operatorname{int}_{1}\left(c l_{2}(F, E)\right)\right) \tilde{\cap} c l_{1}(G, E)=\tilde{\varnothing} .
$$

4) $\Rightarrow$ 3) Let $(F, E) \in \tau_{1},(G, E) \in \tau_{2}$ and $(F, E) \tilde{\cap}(G, E)=\tilde{\varnothing}$. From (4),

$$
c l_{2}\left(\operatorname{int}_{1}\left(c l_{2}(F, E)\right)\right) \tilde{\cap} c l_{1}(G, E)=\tilde{\varnothing} .
$$

Then we have $(F, E) \tilde{\subseteq} \operatorname{int}_{1}\left(c l_{2}(F, E)\right)$ since $(F, E) \tilde{\subseteq} c l_{2}(F, E)$. Therefore,

$$
c l_{2}(F, E) \tilde{\subseteq} c l_{2}\left(\operatorname{int}_{1}\left(c l_{2}(F, E)\right)\right) \text {. }
$$


Hence $c l_{2}(F, E) \tilde{\cap} c l_{1}(G, E)=\tilde{\varnothing}$.

3) $\Rightarrow$ 1) Let $(F, E) \in \tau_{1}$. Then $\tilde{X}-c l_{2}(F, E) \in \tau_{2}$ and $(F, E) \tilde{\cap}\left(\tilde{X}-c l_{2}(F, E)\right)=\tilde{\varnothing}$. From (3),

$$
c l_{2}(F, E) \tilde{\cap} c l_{1}\left(\tilde{X}-c l_{2}(F, E)\right)=\tilde{\varnothing} .
$$

Thus $c l_{2}(F, E) \tilde{\subseteq} \tilde{X}-c l_{1}\left(\tilde{X}-c l_{2}(F, E)\right)=\operatorname{int}_{1}\left(c l_{2}(F, E)\right)$. Therefore, $c l_{2}(F, E) \in \tau_{1}$.

\section{5. $\tau_{2}$-ESDC Soft Topologies $\tau_{1}$}

The family of all semi-open (resp. pre-open, $\alpha$-open, $\beta$-open) soft sets is denoted by $\operatorname{SOS}\left(\tau_{1}\right)$ (resp. $\left.\operatorname{POS}\left(\tau_{1}\right), \alpha O S\left(\tau_{1}\right), \beta O S\left(\tau_{1}\right)\right)$. Also the family of all $\left(\tau_{1}, \tau_{2}\right)$-semi open (resp. $\left(\tau_{1}, \tau_{2}\right)$-pre open, $\left(\tau_{1}, \tau_{2}\right)$ $\alpha$-open, $\left(\tau_{1}, \tau_{2}\right)-\beta$-open) soft sets is denoted by $\operatorname{SOS}\left(\left(\tau_{1}, \tau_{2}\right)\right)$ (resp. $\operatorname{POS}\left(\left(\tau_{1}, \tau_{2}\right)\right), \alpha O S\left(\left(\tau_{1}, \tau_{2}\right)\right)$, $\beta O S\left(\left(\tau_{1}, \tau_{2}\right)\right)$.

Theorem 5.1. If $\mu_{1}=\operatorname{SOS}\left(\left(\tau_{1}, \tau_{2}\right)\right)$ and $\mu_{2}=\operatorname{SOS}\left(\tau_{2}\right), \mu_{1} \tilde{\subseteq} \mu_{2}$ and $(F, E) \in \mu_{1}$ implies $c l_{\mu_{2}}(F, E) \in \mu_{1}$.

Proof. Let $(F, E) \in \mu_{1}$. As $(F, E) \tilde{\subseteq} c l_{2}\left(\right.$ int $\left._{1}(F, E)\right),(F, E) \tilde{\subseteq} c l_{2}\left(\right.$ int $\left._{2}(F, E)\right)$ from Lemma 4.1.

$\mathrm{Cl}_{2}\left(\right.$ int $\left._{1}(F, E)\right)$ is $\mu_{2}$-soft closed for $(F, E) \in S S(X)_{E}$ since

$$
\operatorname{int}_{2}\left(\operatorname{cl}_{2}\left(\operatorname{cl}_{2}\left(\operatorname{int}_{1}(F, E)\right)\right)\right) \tilde{\subseteq} \operatorname{cl}_{2}\left(\operatorname{cl}_{2}\left(\operatorname{int}_{1}(F, E)\right)\right)=\operatorname{cl}_{2}\left(\operatorname{int}_{1}(F, E)\right) .
$$

Hence $(F, E) \tilde{\subseteq} c l_{2}\left(\operatorname{int}_{1}(F, E)\right)$ implies $c l_{\mu_{2}}(F, E) \tilde{\subseteq} c l_{2}\left(i n t_{1}(F, E)\right)$ and

$$
(F, E) \tilde{\subseteq} c l_{\mu_{2}}(F, E) \tilde{\subseteq} c l_{2}\left(i n t_{1}(F, E)\right) \tilde{\subseteq} c l_{2}\left(i n t_{1}\left(c l_{\mu_{2}}(F, E)\right)\right) .
$$

Therefore, we obtain $c l_{\mu_{2}}(F, E) \in \mu_{1}$.

Theorem 5.2. If $\mu_{1}=\beta O S\left(\left(\tau_{1}, \tau_{2}\right)\right)$ and $\mu_{2}=\beta O S\left(\tau_{2}\right), \mu_{1} \tilde{\subseteq} \mu_{2}$ and $(F, E) \in \mu_{1}$ implies $\mathrm{Cl}_{\mu_{2}}(F, E) \in \mu_{1}$.

Proof. Let $(F, E) \in \mu_{1}$. As $(F, E) \tilde{\subseteq} c l_{2}\left(\operatorname{int}_{1}\left(c l_{2}(F, E)\right)\right),(F, E) \tilde{\subseteq} c l_{2}\left(\operatorname{int}_{2}\left(c l_{2}(F, E)\right)\right)$ from Lemma 4.1. $c l_{2}\left(\operatorname{int}_{1}\left(c l_{2}(F, E)\right)\right)$ is $\mu_{2}$-soft closed for $(F, E) \in S S(X)_{E}$ since

$$
\operatorname{int}_{2}\left(\operatorname{cl}_{2}\left(\operatorname{int}_{2}\left(\operatorname{cl}_{2}\left(\operatorname{int}_{1}\left(\operatorname{cl}_{2}(F, E)\right)\right)\right)\right)\right) \tilde{\subseteq} \operatorname{cl}_{2}\left(\operatorname{cl}_{2}\left(\operatorname{int}_{1}\left(\operatorname{cl}_{2}(F, E)\right)\right)\right)=\operatorname{cl}_{2}\left(\operatorname{int}_{1}\left(\operatorname{cl}_{2}(F, E)\right)\right) .
$$

Hence, $(F, E) \tilde{\subseteq} c l_{2}\left(\operatorname{int}_{1}\left(c l_{2}(F, E)\right)\right)$ implies

$$
(F, E) \tilde{\subseteq} c l_{\mu_{2}}(F, E) \tilde{\subseteq} c l_{2}\left(\operatorname{int}_{1}\left(c l_{2}(F, E)\right)\right) \tilde{\subseteq} c l_{2}\left(\operatorname{int}_{1}\left(c l_{2}\left(c l_{\mu_{2}}(F, E)\right)\right)\right) .
$$

Therefore, we obtain $c l_{\mu_{2}}(F, E) \in \mu_{1}$.

\section{Conclusion}

We give the definition of $\left(\tau_{1}, \tau_{2}\right)$-semi open soft (resp. $\left(\tau_{1}, \tau_{2}\right)$-pre open soft, $\left(\tau_{1}, \tau_{2}\right)$ - $\alpha$-open soft, $\left(\tau_{1}, \tau_{2}\right)$ $\beta$-open soft, $\left(\tau_{1}, \tau_{2}\right)^{\prime}$-semi open soft, $\left(\tau_{1}, \tau_{2}\right)^{\prime}$-pre open soft, $\left(\tau_{1}, \tau_{2}\right)^{\prime}-\alpha$-open soft, $\left(\tau_{1}, \tau_{2}\right)^{\prime}-\beta$-open soft) set via two soft topologies. Also we introduce $\left(\tau_{1}, \tau_{2}\right)$-regular open soft and ESDC on two soft topologies. Some properties of some mixed soft operations and characterizations of ESDC are investigated. These properties which are studied are very important for studying anymore.

\section{REFERENCES}

[1] M. I. Ali, F. Feng, X. Liu, W. K. Min and M. Shabir, “On Some New Operations in Soft Set Theory,” Computers \& Mathematics with Applications, Vol. 57, No. 9, 2009, pp. 1547-1553. http://dx.doi.org/10.1016/j.camwa.2008.11.009

[2] S. Hussain and B. Ahmad, "Some Properties of Soft Topological Space," Computers \& Mathematics with Applications, Vol. 62, No. 11, 2011, pp. 4058-4067. http://dx.doi.org/10.1016/j.camwa.2011.09.051 
[3] A. Kandil, O. A. E. Tantawy, S. A. El-Sheikh and A. M. Abd El-Latif, “ $\gamma$-Operation and Decompositions of Some Forms of Soft Continuity in Soft Topological Spaces," Annals of Fuzzy Mathematics and Informatics, 2014, to appear.

[4] P. K. Maji, R. Biswas and A. R. Roy, "Soft Set Theory," Computers \& Mathematics with Applications, Vol. 45, No. 4-5, 2003, pp. 555-562. http://dx.doi.org/10.1016/S0898-1221(03)00016-6

[5] D. Molodtsov, "Soft Set Theory-First Results," Computers \& Mathematics with Applications, Vol. 37, No. 4-5, 1999, pp. 19-31. http://dx.doi.org/10.1016/S0898-1221(99)00056-5

[6] M. Shabir and M. Naz, “On Soft Topological Spaces,” Computers \& Mathematics with Applications, Vol. 61, No. 7, 2011, pp. 1786-1799. http://dx.doi.org/10.1016/j.camwa.2011.02.006

[7] I. Zorlutuna, M. Akdag, W. K. Min and S. Atmaca, "Remarks on Soft Topological Spaces,” Annals of Fuzzy Mathematics and Informatics, Vol. 3, No. 2, 2012, pp. 171-185. 